\section{A) Check for updates}

Cite this: Inorg. Chem. Front., 2019, 6,631

DOI: 10.1039/c9qi90006c

rsc.li/frontiers-inorganic

\title{
Correction: High-performance alkaline hydrogen evolution electrocatalyzed by a $\mathrm{Ni}_{3} \mathrm{~N}-\mathrm{CeO}_{2}$ nanohybrid
}

\author{
Zhaomei Sun, ${ }^{\mathrm{a}, \mathrm{b}}$ Jiayu Zhang, ${ }^{\mathrm{b}}$ Junfeng Xie, ${ }^{\mathrm{a}}$ Xiangjiang Zheng, ${ }^{\mathrm{b}}$ Min Wang, ${ }^{\mathrm{b}}$ \\ Xuemei Li*b and Bo Tang*a
}

Correction for 'High-performance alkaline hydrogen evolution electrocatalyzed by a $\mathrm{Ni}_{3} \mathrm{~N}-\mathrm{CeO}_{2}$ nanohybrid' by Zhaomei Sun et al., Inorg. Chem. Front., 2018, 5, 3042-3045.

The authors regret errors within the affiliations listed for authors Zhaomei Sun and Junfeng Xie. The corrected list of author affiliations for this article is as shown above.

The Royal Society of Chemistry apologises for these errors and any consequent inconvenience to authors and readers.

\footnotetext{
${ }^{a}$ College of Chemistry, Chemical Engineering and Materials Science, Collaborative Innovation Center of Functionalized Probes for Chemical Imaging in Universities of Shandong, Key Laboratory of Molecular and Nano Probes, Ministry of Education, Institute of Molecular and Nano Science, Shandong Normal University, Jinan 250014, Shandong, China. E-mail: tangb@sdnu.edu.cn

${ }^{b}$ Shandong Provincial Key Laboratory of Detection Technology for Tumor Markers, School of Chemistry and Chemical Engineering, Linyi University, Linyi 276005, Shandong, China.E-mail:xuemei_li@yeah.net
} 